\title{
The Antonov problem for rotating systems
}

\author{
A. De Martino, E.V. Votyakov D.H.E. Gross \\ Hahn-Meitner-Institut, Bereich Theoretische Physik \\ Glienickerstr. 100, 14109 Berlin (Germany)
}

\begin{abstract}
We study the classical Antonov problem (of retrieving the statistical equilibrium properties of a self-gravitating gas of classical particles obeying Boltzmann statistics in space and confined in a spherical box) for a rotating system. It is shown that a critical angular momentum $\lambda_{c}$ (or, in the canonical language, a critical angular velocity $\omega_{c}$ ) exists, such that for $\lambda<\lambda_{c}$ the system's behaviour is qualitatively similar to that of a non-rotating gas, with a high energy disordered phase and a low energy collapsed phase ending with Antonov's limit, below which there is no equilibrium state. For $\lambda>\lambda_{c}$, instead, the low-energy phase is characterized by the formation of two dense clusters (a "binary star"). Remarkably, no Antonov limit is found for $\lambda>\lambda_{c}$. The thermodynamics of the system (phase diagram, caloric curves, local stability) is analyzed and compared with the recently-obtained picture emerging from a different type of statistics which forbids particle overlapping.
\end{abstract}

\section{Introduction}

The name "Antonov problem" is usually referred to the study of the meanfield equilibrium state of self-gravitating $N$-body systems of classical particles in a finite volume. One considers the system with Hamiltonian

$$
\begin{gathered}
H_{N} \equiv H_{N}\left(\left\{\boldsymbol{r}_{i}\right\},\left\{\boldsymbol{p}_{i}\right\}\right)=\frac{1}{2} \sum_{i=1}^{N} p_{i}^{2}+\Phi\left(\left\{\boldsymbol{r}_{i}\right\}\right) \\
\Phi\left(\left\{\boldsymbol{r}_{i}\right\}\right)=-G \sum_{1 \leq i<j \leq N} \frac{1}{\left|\boldsymbol{r}_{i}-\boldsymbol{r}_{j}\right|}
\end{gathered}
$$

enclosed in a finite three-dimensional spherical box of radius $R$, which is necessary in order to prevent particles from evaporating. In $(1,2): \boldsymbol{r}_{i} \in V \subset \mathbb{R}^{3}$ and $\boldsymbol{p}_{i} \in \mathbb{R}^{3}$ denote, respectively, the position and momentum of the $i$-th particle, $G$ is the gravitational constant, and $V$ stands for the (volume of the) box containing the particles; we set the particles' masses to one. The main problems are: (a) find the equilibrium configurations, i.e. the particle density 
profiles $\rho(\boldsymbol{r})\left(\int_{V} \rho(\boldsymbol{r}) d \boldsymbol{r}=N\right)$ that maximize the microcanonical entropy $(\alpha=$ constant)

$$
S_{N} \equiv S_{N}(E)=\log \left[\frac{\alpha}{N !} \int \delta\left(H_{N}-E\right) D \boldsymbol{r} D \boldsymbol{p}\right]
$$

$\left(D \boldsymbol{x}=\prod_{i=1}^{N} d \boldsymbol{x}_{i}\right)$; and (b) analyze the corresponding thermodynamics. Clearly, the existence of such profiles and their actual structure will depend on the value of the total energy $E$. Intuitively, when $E$ is large and positive (that is when the kinetic term dominates) a gaseous-type of structure will be preferred, with particles uniformly spread over $V$, whereas when $E$ is sufficiently negative particles are expected to attract each other in a dense core embedded in a vapor. The situation presents however several subtleties. Assuming that particles are point masses obeying Boltzmann statistics in space, i.e. that they can come arbitrarily close to each other, it is found within the mean-field approximation [1-5] that

(a) there is no global entropy maximum, therefore all maxima are local and correspond to metastable situations;

(b) if $\frac{R E}{G N^{2}} \equiv \epsilon>\epsilon_{c} \simeq-0.335$ (i.e. at sufficiently high energies, for we take $R$ to be fixed) there exist local entropy extrema where the particle density satisfies the condition

$$
\rho(\boldsymbol{r})=A \exp \left[\beta \int \frac{\rho\left(\boldsymbol{r}^{\prime}\right)}{\left|\boldsymbol{r}-\boldsymbol{r}^{\prime}\right|} d \boldsymbol{r}^{\prime}\right] \quad \beta=\frac{3 / 2}{\left[E-\Phi\left(\left\{\boldsymbol{r}_{i}\right\}\right)\right]}
$$

where $A$ is a constant. Notice that $\beta$ is itself a function of $\rho$. The physically admissible solutions of (4) are spherically symmetric (in fact, they can be mapped onto the solutions of the Emden equation $y^{\prime \prime}+2 y^{\prime} / x=e^{-y}$, with $y(0)=y^{\prime}(0)=0$ ) and are called microcanonical "isothermal spheres";

(c) in general, local extrema in the space of $\rho$ 's can be maxima (metastable states) or saddle points (unstable). Microcanonical isothermal spheres are local maxima if the "density contrast" $\rho(0) / \rho(R) \lesssim 709$, otherwise they are at most saddle points of the entropy surface;

(d) if $\epsilon$ lies in the range between -0.2 and $\epsilon_{c}$ isothermal spheres have negative specific heat ${ }^{1}$ : in such states the system heats up and shrinks upon decreasing energy;

(e) finally, if $\epsilon<\epsilon_{c}$ (i.e. at sufficiently low energies) there are not even local entropy extrema.

The standard interpretation [2] is that, while at high energies an equilibrium configuration is achievable in the form of an isothermal sphere, below $\epsilon_{c}$ the system collapses to a dense core and overheats, following the negative-specificheat trend to its extreme consequences. Such a "transition", occurring at the

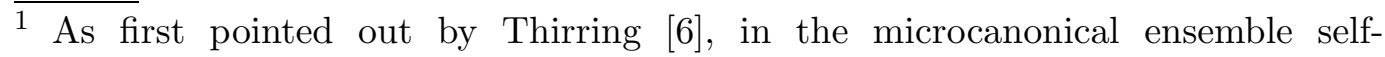
gravitating systems can have negative specific heat, at odds with the canonical setup, in which it is related to energy fluctuations and positive definite. 
Antonov point $\epsilon=\epsilon_{c}$, is known as "gravothermal collapse" or "catastrophe" (see also $[7,8]$ for a more formal treatment of this transition).

In this work we extend the above theory to rotating systems, by including the effects of the angular momentum $\boldsymbol{L}=\sum_{i=1}^{N} \boldsymbol{r}_{i} \times \boldsymbol{p}_{i}$ as a second conserved quantity besides $E$, and discuss the thermodynamics and the corresponding equilibrium states (which will now depend on both $E$ and $\boldsymbol{L}$ ). Our work is motivated mainly by the crucial role rotation is expected to play in astrophysical systems on different scales such as globular clusters and the cores of elliptic galaxies $[9,10]$, both of which can be modeled by $(1,2)$. (Hence our "particles" can be interpreted equally well as atoms and as stars.) A gravitydriven collapse can be seen as the prime cause for the equilibrium shaping of self-gravitating systems, both rotating and not. But the nature and the result of this collapse are expected to change significantly in the presence of rotation, and in particular for systems rotating sufficiently fast. Rotating systems were tackled in the past by different models (see e.g. [11,12] for a discussion of the stability of rotating gaseous cylinders, and the more recent works $[13,14]$ and references therein), but a complete analysis of the Antonov problem with angular momentum is still lacking. Some progress was achieved recently for another class of systems where particles are assumed to obey a statistics that prohibits overlapping, the so-called Lynden-Bell statistics $[15,16]$.

Indeed, we will show that the above picture (a)-(e) holds with minor modifications for slowly rotating systems, that is when $|\boldsymbol{L}|=L$ is sufficiently small. In particular, defining the reduced angular momentum $\lambda=L / \sqrt{R G N^{3}}$, we identify an Antonov line $\epsilon_{c}(\lambda)$, with $\epsilon_{c}(0)=\epsilon_{c} \simeq-0.335$ which plays in rotating systems the same role as the Antonov point plays in non-rotating ones. For $\lambda$ larger than a critical value $\lambda_{c} \simeq 0.45$, however, the collapse occurs not to a single dense cluster but to a double cluster (a "binary star"), signaling a breaking of the (axial-)rotational symmetry of (1). It is remarkable that, strictly speaking, for $\lambda>\lambda_{c}$ there is no Antonov limit: double cluster solutions exist at all energies, albeit being locally unstable (they are saddle points of entropy in the space of $\rho$ 's). The lower the energy, the more dense and point-like the two clusters. We calculate the global phase diagram of the model in the $(\epsilon, \lambda)$ plane and analyze the thermodynamics by studying the phase transition that take place and the caloric curves. The equilibrium density profiles obtained in the different regions of the phase diagram show that, depending on the values of $\epsilon$ and $\lambda$, the formation of "rings", "single stars", "double stars" and "disk"-like structures is possible.

From a broad statistical mechanics viewpoint, it is well known that selfgravitating systems, and more generally systems with long-range interaction 
potentials $^{2}$, represent a subtle and delicate issue [17]. For a recent critical discussion of the general statistical mechanics of non-trivial systems see [18]. The main problem is that the usual thermodynamic limit $(N \rightarrow \infty, V \rightarrow$ $\infty, N / V$ finite) for systems with potentials such as (2) does not exist [19]. Their thermodynamics is evidently not extensive. Now extensive systems necessarily have positive specific heat, but this need not be true for non-extensive ones [6, 20-24]. Hence the canonical and microcanonical description of nonextensive systems do not necessarily coincide (they do only in "regular" phases with positive specific heat, and in the dilute limit $\left(N \rightarrow \infty, V \rightarrow \infty, N / V^{1 / 3}\right.$ finite) discussed at length in $[25,26])$. In the most general and physically interesting cases, self-gravitating systems should be studied in the microcanonical ensemble, a circumstance that obviously introduces several technical difficulties which are amplified by the introduction of rotation.

The choice of Boltzmann statistics, which is made by most authors [4], to describe the behaviour of particles contributes to complicate the situation further. The basic assumption is that all particles can eventually occupy the same point/cell in space, i.e. overlapping between particles is admitted. With Boltzmann statistics, the system (1,2) has no ground state, hence the entropy is not bounded. This is the ultimate origin of Antonov's catastrophe, even (as we shall see) in rotating systems. Clearly, if one wants to describe a system where Newtonian gravity is the dominant macroscopic interaction then configurations with high particle density should be avoided, for at such short distances quantum effects and nuclear interactions become more important than gravity. One way around this problem is to introduce hard cores for particles. Another substantially equivalent way is to use Lynden-Bell statistics [27] instead of Boltzmann's.

We shall proceed as follows. In Sec. 2, we will expound our microcanonical mean-field theory, deriving the analogous of Eq. (4) for rotating systems. In Sec. 3 we will show results from the numerical solution of the new equation, and discuss in particular the phase diagram in the $(\epsilon, \lambda)$ plane and the caloric curves. A comparison between the Lynden-Bell scenario discussed in $[15,16]$ and the Boltzmann theory is presented in Sec. 4. Finally, we formulate our conclusions with some final remarks .

\section{Mean-field theory}

The theoretical analysis of Antonov's problem in a rotating framework can be carried out along the lines of the mean-field approximation described in $[15,16]$,

2 By which we mean potentials decaying with the interparticle distance $r$ more slowly than $r^{-D-\delta}$ with $\delta>0$ in $D$ dimensions when $r \rightarrow \infty$. 
where the equilibrium properties of a self-gravitating and rotating system were studied assuming Lynden-Bell statistics for particles ${ }^{3}$. The microcanonical entropy in our case is given by

$$
S_{N} \equiv S_{N}(E, \boldsymbol{L})=\log \left[\frac{\alpha}{N !} \int \delta\left(H_{N}-E\right) \delta\left(\boldsymbol{L}-\sum_{i=1}^{N} \boldsymbol{r}_{i} \times \boldsymbol{p}_{i}\right) D \boldsymbol{r} D \boldsymbol{p}\right]
$$

where $\alpha$ is a constant that makes the argument of the logarithm dimensionless. The integral over momenta, namely

$$
F_{N}\left(\left\{\boldsymbol{r}_{i}\right\}, K, \boldsymbol{L}\right)=\int \delta\left(K-\frac{1}{2} \sum_{i=1}^{N} p_{i}^{2}\right) \delta\left(\boldsymbol{L}-\sum_{i=1}^{N} \boldsymbol{r}_{i} \times \boldsymbol{p}_{i}\right) D \boldsymbol{p}
$$

can be calculated via its Laplace transform (integrals resulting from insertion of the integral representation of the $\delta$-function are at most of Gaussian type, hence trivial $[15,16,28])$

$$
\begin{aligned}
\widetilde{F}_{N}\left(\left\{\boldsymbol{r}_{i}\right\}, s, \boldsymbol{L}\right) & =\int_{0}^{\infty} F_{N}\left(\left\{\boldsymbol{r}_{i}\right\}, K, \boldsymbol{L}\right) e^{-s K} d K \quad(\Re s>0) \\
& =(\operatorname{det} \mathbb{I})^{-1 / 2}\left(\frac{2 \pi}{s}\right)^{\frac{3 N-3}{2}} e^{-\frac{1}{2} s \boldsymbol{L}^{T} \mathbb{I}^{-1} \boldsymbol{L}}
\end{aligned}
$$

where $\mathbb{I} \equiv \mathbb{I}\left(\left\{\boldsymbol{r}_{i}\right\}\right)$ denotes the inertia tensor, with elements $(a, b=1,2,3)$

$$
I_{a b}\left(\left\{\boldsymbol{r}_{i}\right\}\right)=\sum_{i=1}^{N}\left(r_{i}^{2} \delta_{a b}-r_{i, a} r_{i, b}\right)
$$

and $\boldsymbol{L}^{T} \mathbb{I}^{-1} \boldsymbol{L}=\sum_{a, b} L_{a} I_{a b}^{-1} L_{b}$. Inversion of (8) gives

$$
F_{N}\left(\left\{\boldsymbol{r}_{i}\right\}, K, \boldsymbol{L}\right)= \begin{cases}\frac{(2 \pi)^{\frac{3 N-3}{2}}}{\Gamma\left(\frac{3 N-3}{2}\right) \sqrt{\operatorname{det} \mathbb{I}}}\left(K-\frac{1}{2} \boldsymbol{L}^{T} \mathbb{I}^{-1} \boldsymbol{L}\right)^{\frac{3 N-5}{2}} & K>\frac{1}{2} \boldsymbol{L}^{T} \mathbb{I}^{-1} \boldsymbol{L} \\ 0 & \text { otherwise }\end{cases}
$$

hence one is left with

$$
S_{N}=\log \left[\frac{\alpha A}{N !} \int \frac{\left[E-\frac{1}{2} \boldsymbol{L}^{T} \mathbb{I}^{-1} \boldsymbol{L}-\Phi\left(\left\{\boldsymbol{r}_{i}\right\}\right)\right]^{\frac{3 N-5}{2}}}{\sqrt{\operatorname{det} \mathbb{I}}} D \boldsymbol{r}\right]
$$

where $A=(2 \pi)^{\frac{3 N-3}{2}} / \Gamma((3 N-3) / 2)$. If one is interested (as we are) in the large $N$ limit, one can keep only the terms of dominating order in $N$ in (11).

3 A calculation similar to the one presented here (with Boltzmann statistics) was performed in [28], where however the resulting stationarity condition is studied in the context of the Thirring model [6], a simplified model of a rotating self-gravitating gas. 
This yields

$$
S_{N}=\log \left[\frac{\alpha A}{N !} \int\left[E-\frac{1}{2} \boldsymbol{L}^{T} \mathbb{I}^{-1} \boldsymbol{L}-\Phi\left(\left\{\boldsymbol{r}_{i}\right\}\right)\right]^{\frac{3 N}{2}} D \boldsymbol{r}\right]
$$

and it remains to integrate over $V^{N}$.

To this aim, we partition the spherical box $V$ into $K$ identical cells labeled by the positions $\boldsymbol{r}_{k}$ of their centers $(k=1, \ldots, K)$, and we let $n\left(\boldsymbol{r}_{k}\right)$ denote the number of particles inside the cell at position $\boldsymbol{r}_{k}$, with $\sum_{k=1}^{K} n\left(\boldsymbol{r}_{k}\right)=N$. In order to turn the integral into a sum over the cells, we adopt the following mean-field approximation for the potential $\Phi$ and the components of the inertia tensor as functionals of the density profile $\rho \equiv \rho(\boldsymbol{r})=(K / V) n(\boldsymbol{r})$ :

$$
\begin{gathered}
\Phi\left(\left\{\boldsymbol{r}_{i}\right\}\right) \rightarrow \Phi[\rho]=-\frac{G}{2} \int \frac{\rho(\boldsymbol{r}) \rho\left(\boldsymbol{r}^{\prime}\right)}{\left|\boldsymbol{r}-\boldsymbol{r}^{\prime}\right|} d \boldsymbol{r} d \boldsymbol{r}^{\prime} \\
I_{a b}\left(\left\{\boldsymbol{r}_{i}\right\}\right) \rightarrow I_{a b}[\rho]=\int \rho(\boldsymbol{r})\left(r^{2} \delta_{a b}-r_{a} r_{b}\right) d \boldsymbol{r}
\end{gathered}
$$

This choice automatically excludes correlations from the theory. The microcanonical mean-field entropy can now be written as

$$
S_{N}^{\mathrm{mf}}=\log \left[\frac{\alpha A}{N !} \int\left[E-\frac{1}{2} \boldsymbol{L}^{T} \mathbb{I}^{-1} \boldsymbol{L}-\Phi[\rho]\right]^{\frac{3 N}{2}} P[\rho] d \rho(\boldsymbol{r})\right]
$$

where $P[\rho]$ is the probability to observe a density profile $\rho$. Now $P[\rho]$ is just proportional to the number of ways in which our $N$ particles can be distributed into the $K$ cells, and is thus determined by the statistical model by which one describes the behaviour of the particles. Assuming Boltzmann statistics, there is no spatial constraint for particles so that all of them can eventually occupy the same cell in $V$. This leads to

$$
P[\rho] \propto \frac{N !}{n\left(\boldsymbol{r}_{1}\right) ! \cdots n\left(\boldsymbol{r}_{K}\right)} \simeq N ! \exp \left[-\frac{N K}{V} \int c(\boldsymbol{r})(\log c(\boldsymbol{r})-1) d \boldsymbol{r}\right]
$$

where in obtaining the last expression we used Stirling's approximation and defined the relative cell occupancy $c(\boldsymbol{r})=n(\boldsymbol{r}) / N=V \rho(\boldsymbol{r}) /(K N)$. Introducing the dimensionless variable $\boldsymbol{x}=\boldsymbol{r} / R$ and neglecting irrelevant constants, one finally arrives at

$$
\begin{gathered}
S_{N}^{\mathrm{mf}}=\log \int e^{N \Sigma[c]} d c(\boldsymbol{x}) \\
\Sigma[c]=\frac{3}{2} \log \left[E-\frac{1}{2} \boldsymbol{L}^{T} \mathbb{I}^{-1} \boldsymbol{L}-\Phi[c]\right]-\frac{1}{\Theta} \int c(\boldsymbol{x})(\log c(\boldsymbol{x})-1) d \boldsymbol{x}
\end{gathered}
$$

where $\Theta=\int c(\boldsymbol{x}) d \boldsymbol{x}=\frac{V}{K R^{3}}$.

For $N$ large though finite, $S_{N}^{\mathrm{mf}}$ can be estimated by the steepest descent 
method, which for $S=S_{N}^{\mathrm{mf}} / N$ yields

$$
S=\max _{c(\boldsymbol{x})}\left[\frac{3}{2} \log \left[E-\frac{1}{2} \boldsymbol{L}^{T} \mathbb{I}^{-1} \boldsymbol{L}-\Phi[c]\right]-\frac{1}{\Theta} \int c(\boldsymbol{x})(\log c(\boldsymbol{x})-1) d \boldsymbol{x}\right]
$$

Notice that the stationarity properties of $S_{N}$ do not depend on $\Theta$. This is most easily seen by setting $f(\boldsymbol{x})=c(\boldsymbol{x}) / \Theta$, which leads to

$$
S=\max _{f(\boldsymbol{x})}\left[\frac{3}{2} \log \left[E-\frac{1}{2} \boldsymbol{L}^{T} \mathbb{I}^{-1} \boldsymbol{L}-\Phi[f]\right]-\int f(\boldsymbol{x})(\log f(\boldsymbol{x})-1) d \boldsymbol{x}+\log \Theta\right]
$$

We remark that $\mathbb{I} \equiv \mathbb{I}[f]$. In terms of $f$ and in units of $G N^{2} / R$ and $N R^{2}$, respectively, $\Phi$ and $I_{a b}$ (see (13),(9)) become simply

$$
\begin{aligned}
\Phi[f] & =-\frac{1}{2} \int \frac{f(\boldsymbol{x}) f\left(\boldsymbol{x}^{\prime}\right)}{\left|\boldsymbol{x}-\boldsymbol{x}^{\prime}\right|} d \boldsymbol{x} d \boldsymbol{x}^{\prime} \\
I_{a b}[f] & =\int f(\boldsymbol{x})\left(x^{2} \delta_{a b}-x_{a} x_{b}\right) d \boldsymbol{x}
\end{aligned}
$$

The stationarity condition $\delta S / \delta f=0$ implies the following equation for the extrema of the entropy:

$$
f(\boldsymbol{x})=\exp \left[\beta \int \frac{f\left(\boldsymbol{x}^{\prime}\right)}{\left|\boldsymbol{x}-\boldsymbol{x}^{\prime}\right|} d \boldsymbol{x}^{\prime}+\frac{\beta}{2}(\boldsymbol{\omega} \times \boldsymbol{x})^{2}+\mu\right]
$$

where $\boldsymbol{\omega}=\mathbb{I}^{-1} \boldsymbol{L}$ is the angular velocity and $\mu$ is a Lagrange multiplier that ensures the constraint $\int f(\boldsymbol{x}) d \boldsymbol{x}=1$, and

$$
\beta=\frac{3 / 2}{\left[E-\frac{1}{2} \boldsymbol{L}^{T} \mathbb{I}^{-1} \boldsymbol{L}-\Phi[f]\right]}
$$

is the inverse kinetic energy. Notice that $\beta \equiv \beta[f]$.

Eq. (23) is the analogous of (4) for a rotating system. Unfortunately, it cannot be solved analytically. However, some simple qualitative considerations are possible. In absence of rotation, the solution of (23) is known to depend only on $x=|\boldsymbol{x}|$. The minimum expected effect of rotation is to create distortions that cannot be described by $x$ alone. Hence it is necessary to introduce angular variables, and it is convenient to pass to spherical coordinates $(x, \theta, \phi)$. If the system is slowly rotating and if, as we shall assume in the following, the angular momentum is parallel to the 3 -axis, one may neglect the $\phi$-dependence and write formally the solution of (23) as a sum of Legendre polynomials weighted by functions of $x$ alone $^{4}$ :

$$
f(\boldsymbol{x})=\sum_{l=0}^{\infty} f_{l}(x) P_{l}(\cos \theta)
$$

$\overline{4}$ When in the following we speak of axial rotational symmetry or for short of axial symmetry we refer always to the 3 -axis. 
This procedure is well-known [29], and has been recently applied to the Antonov problem for slowly rotating systems by Chavanis [14]. The latter, however, do not display any serious qualitative difference from non rotating ones. The most interesting phenomena occur at high angular momenta, and in order to describe them it is necessary to include the dependence on $\phi$. We thus write the solution of (23) as

$$
f(\boldsymbol{x})=\sum_{l=0}^{\infty} \sum_{m=-l}^{l} f_{l, m}(x) Y_{l m}(\theta, \phi)
$$

where $Y_{l m}$ are real spherical harmonics and $f_{l, m}$ are their weights (again functions of $x$ alone), which will have to be determined. Clearly, this expansion differs from the one in Legendre polynomials essentially for the terms with $m \neq 0$. We shall see that these terms are the ones that provide the relevant new physics at high angular momenta.

Using this expansion it is easy to show the following statements. The normalization $\int f(\boldsymbol{x}) d \boldsymbol{x}=1$ is equivalent to

$$
2 \sqrt{\pi} \int x^{2} f_{0,0}(x) d x=1
$$

so that the $(0,0)$ harmonics is ensures this condition. The coordinates of the center of mass are given by

$$
\begin{aligned}
x_{1}^{C M} & \equiv \int x_{1} f(\boldsymbol{x}) d \boldsymbol{x}=2 \sqrt{\frac{\pi}{3}} \int x^{3} f_{1,1}(x) d x \\
x_{2}^{C M} & \equiv \int x_{2} f(\boldsymbol{x}) d \boldsymbol{x}=2 \sqrt{\frac{\pi}{3}} \int x^{3} f_{1,-1}(x) d x \\
x_{3}^{C M} & \equiv \int x_{3} f(\boldsymbol{x}) d \boldsymbol{x}=2 \sqrt{\frac{\pi}{3}} \int x^{3} f_{1,0}(x) d x
\end{aligned}
$$

so that harmonics with $l=1$ are responsible for fixing the center of mass. It is convenient to set the latter in the center of the spherical box $(\boldsymbol{x}=\mathbf{0})$, which amounts to postulating $f_{1, m}=0$ for $m \in\{-1,0,1\}$. The diagonal components of the inertia matrix read ${ }^{5}$

$$
\begin{gathered}
I_{11}=\sqrt{\frac{16 \pi}{9}} \int x^{2} f_{0,0}(x) d x+\sqrt{\frac{4 \pi}{45}} \int x^{4} f_{2,0}(x) d x-\sqrt{\frac{4 \pi}{15}} \int x^{4} f_{2,2}(x) d x \\
I_{22}=\sqrt{\frac{16 \pi}{9}} \int x^{2} f_{0,0}(x) d x+\sqrt{\frac{4 \pi}{45}} \int x^{4} f_{2,0}(x) d x+\sqrt{\frac{4 \pi}{15}} \int x^{4} f_{2,2}(x) d x \\
I_{33}=\sqrt{\frac{16 \pi}{9}} \int x^{2} f_{0,0}(x) d x-\sqrt{\frac{16 \pi}{45}} \int x^{4} f_{2,0}(x) d x
\end{gathered}
$$

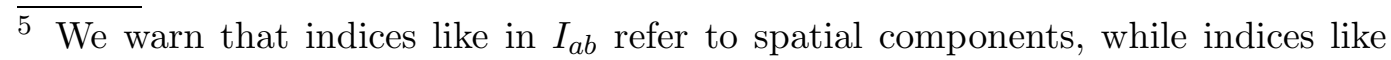
$f_{l, m}$ refer to harmonics. 
while for the off-diagonal elements one gets

$$
\begin{aligned}
& I_{12}=-\sqrt{\frac{4 \pi}{15}} \int x^{4} f_{2,-2}(x) d x \\
& I_{13}=-\sqrt{\frac{4 \pi}{15}} \int x^{4} f_{2,1}(x) d x \\
& I_{23}=-\sqrt{\frac{4 \pi}{15}} \int x^{4} f_{2,-1}(x) d x
\end{aligned}
$$

In particular, one has

$$
I_{11}-I_{22}=-\sqrt{\frac{16 \pi}{15}} \int x^{4} f_{2,2}(x) d x
$$

so that this parameter, which will play a foremost role in the following analysis, only depends on the $(2,2)$ harmonics.

Using spherical harmonics as a basis set, for the Newtonian potential one has $[30,31]$ :

$$
\frac{1}{\left|\boldsymbol{x}-\boldsymbol{x}^{\prime}\right|}=\sum_{l=0}^{\infty} \sum_{m=-l}^{l} \frac{4 \pi}{2 l+1} \frac{\min \left\{x, x^{\prime}\right\}^{l}}{\max \left\{x, x^{\prime}\right\}^{l+1}} Y_{l m}(\theta, \phi) Y_{l m}\left(\theta^{\prime}, \phi^{\prime}\right)
$$

Now it is easy to see that the so far unknown functions $f_{l, m}$ satisfy the following integral equation (which can be found by multiplying both sides of (23) by $Y_{l m}$ and integrating over angular variables):

$$
f_{l, m}(x)=\int Y_{l m}(\theta, \phi) e^{\beta \sum_{l^{\prime}, m^{\prime}} u_{l^{\prime}, m^{\prime}}(x) Y_{l^{\prime} m^{\prime}}(\theta, \phi)+\frac{1}{2} \beta \omega^{2} x^{2} \sin ^{2} \theta} d \theta d \phi
$$

with

$$
u_{l, m}(x)=\frac{4 \pi}{2 l+1} \int \frac{\min \left\{x, x^{\prime}\right\}^{l}}{\max \left\{x, x^{\prime}\right\}^{l+1}} f_{l, m}\left(x^{\prime}\right)\left(x^{\prime}\right)^{2} d x^{\prime}
$$

Despite their unfriendly look, Eqs $(37,38)$, which should be solved together in a self-consistent way, can be dealt with numerically. Technical details are similar to those described in $[15,16]$ in relation to the Lynden-Bell statistics case, and we will skip them here. Let it suffice to say that for the computation the series (36) was truncated at the order $l_{\max }=12$ and that all harmonics (even and odd) up to $l_{\max }$ were included, except those with $l=1$. Notice that this does not imply a truncation of the expansion (26), i.e. the $f_{l, m}$ exist also for orders higher than $l_{\max }$. The effects of truncating the potential are wellknown, and are discussed e.g. in [16]. In short, when truncated at higher and higher order the series (36) differs more and more from the real potential in a narrower and narrower region around $\boldsymbol{x}=\boldsymbol{x}^{\prime}$, in a way that is reminiscent of the Gibbs phenomenon. As we said, the angular momentum was taken to lie along the 3 -direction. In this setup, solutions of (23) were calculated as 
functions of $\epsilon=\frac{E R}{G N^{2}}$ and $\lambda=\frac{L}{\sqrt{R G N^{3}}}$; results of this analysis are given and discussed in the next section.

\section{Results}

\subsection{Relevant quantities}

The crucial quantity in the analysis that will follow is given by

$$
\operatorname{Hes}(\epsilon, \lambda)=\operatorname{det}\left(\begin{array}{cc}
\partial_{\epsilon}^{2} S & \partial_{\lambda} \partial_{\epsilon} S \\
\partial_{\epsilon} \partial_{\lambda} S & \partial_{\lambda}^{2} S
\end{array}\right)=\kappa_{1} \kappa_{2}
$$

where $\kappa_{1,2}$ are the eigenvalues of the matrix, ordered assuming $\kappa_{1}>\kappa_{2}$. The relevance of $\operatorname{Hes}(\epsilon, \lambda)$ lies in the fact that it relates the thermostatistical equilibrium properties of systems in the microcanonical ensemble to the topology of the entropy surface in the space of conserved macroscopic quantities. In general, it can be shown $[17,32]$ that pure, stable thermodynamic phases with positive heat capacity are characterized by having $\kappa_{1}<0$ (hence $\operatorname{Hes}(\epsilon, \lambda)>0$ ). Here, $S$ as a function of both $\epsilon$ and $\lambda$ is concave, and statistical ensembles (microcanonical and canonical) are equivalent. When at least $\kappa_{1}>0$, one has a phase coexistence region with negative specific heat, and statistical ensembles are inequivalent. $S(\epsilon, \lambda)$ has a convex part that is ignored by the canonical ensemble, which considers the concave hull of $S$. The convex region corresponds to negative specific heat. For self-gravitating systems, (39) provides a powerful characterization of some of the relevant quantities. In particular, we shall concentrate on the following aspects of the phase diagram.

Antonov limit. For each fixed $\lambda$, the Antonov limit $\epsilon_{c}(\lambda)$ is the minimum $\epsilon$ for which a solution of (23) exists. In general, $\epsilon_{c}(\lambda)$ can be defined by the condition

$$
\lim _{\epsilon \downarrow \epsilon_{c}(\lambda)} \kappa_{1}=+\infty
$$

(Strictly speaking, $\kappa_{1}$ can diverge only in the mean-field approximation in the $N \rightarrow \infty$ limit at fixed $\epsilon$ and $\lambda$.) This is a straightforward generalization of the non-rotating case, where at the Antonov point $\epsilon_{c} \equiv \epsilon_{c}(0)$ one has

$$
\lim _{\epsilon \downarrow \epsilon_{c}} \frac{\partial \beta}{\partial \epsilon}=+\infty
$$

When two (or more) conserved quantities have to be taken into consideration, the above condition becomes a condition on the maximum eigenvalue of the matrix of second derivatives of $S$ with respect to the conserved quantities. 
Upon varying $\lambda$, the Antonov limit $\epsilon_{c}(\lambda)$ describes a line in the $(\epsilon, \lambda)$ plane. We call this line the Antonov line.

Phase coexistence. By virtue of the properties of (39), starting in the highenergy phase and decreasing $\epsilon$ at fixed $\lambda$ one finds the beginning of a phase coexistence region when

$$
\operatorname{Hes}(\epsilon, \lambda)=0
$$

is fulfilled. We shall denote by $\epsilon_{0}(\lambda)$ the corresponding value of $\epsilon$. In particular, at $\epsilon_{0}(\lambda)$ the specific heat at constant $\beta \omega=-\frac{\partial S}{\partial \lambda}(\omega=|\boldsymbol{\omega}|)$, i.e.

$$
c_{\beta \omega}=-\frac{\beta^{2}}{\operatorname{Hes}(\epsilon, \lambda)} \frac{\partial^{2} S}{\partial \lambda^{2}}
$$

has a pole and changes sign (from positive to negative) upon decreasing $\epsilon$. Again, varying $\lambda$ the point $\epsilon_{0}(\lambda)$ describes a line in the $(\epsilon, \lambda)$ plane (with $\left.\epsilon_{0}(0) \simeq-0.2\right)$, which separates a pure thermodynamic phase from a phase coexistence region.

Bifurcation to axially asymmetric solutions. For sufficiently high $\lambda$, solutions of (23) without axial rotational symmetry ("binary stars") bifurcate (continuously) from axially-symmetric solutions $[15,16]$. This bifurcation can be detected with the aim of the order parameter (see (35))

$$
D=\left|I_{11}-I_{22}\right|=\left|\sqrt{\frac{16 \pi}{15}} \int x^{4} f_{2,2}(x) d x\right|
$$

introduced in [15]. It is easy to understand that solutions with $D=0$ are axially-symmetric, while solutions with $D \neq 0$ break axial rotational symmetry. If $\lambda=0$ the system is necessarily isotropic $\left(I_{11}=I_{22}=I_{33}\right)$ and spherical symmetry cannot be broken. This reflects the fact that the solution of (23) depends only on $x$, so that the series expansion (26) is truncated after the first term, which involves only the $Y_{00}$ harmonics. When $\lambda \neq 0$, anisotropies may occur $\left(I_{33} \neq I_{11}, I_{22}\right)$ and one can have either solutions that are rotationallysymmetric around the 3 -axis $\left(I_{11}=I_{22}\right.$ or $\left.D=0\right)$, which eventually depend on $x$ and $\theta$ to account for obvious rotation-induced distortions, or solutions that are asymmetric around the 3 -axis $\left(I_{11} \neq I_{22}\right.$ or $\left.D \neq 0\right)$. The latter must depend on $x, \theta$ and $\phi$, and correspond to double clusters. However, it turns out that such solutions appear only when $\lambda$ is larger than a threshold value and $\epsilon$ is sufficiently low (see also the discussion at the end of Sec. 4, and in particular Fig. 5).

Stability of the double cluster solutions. Finally, we shall be interested in the local stability properties of double-cluster solutions (high- $\epsilon$, axially symmetric solutions are locally stable because in this limit the kinetic term of (1) dominates and the system behaves more and more as an ideal gas). This amounts 


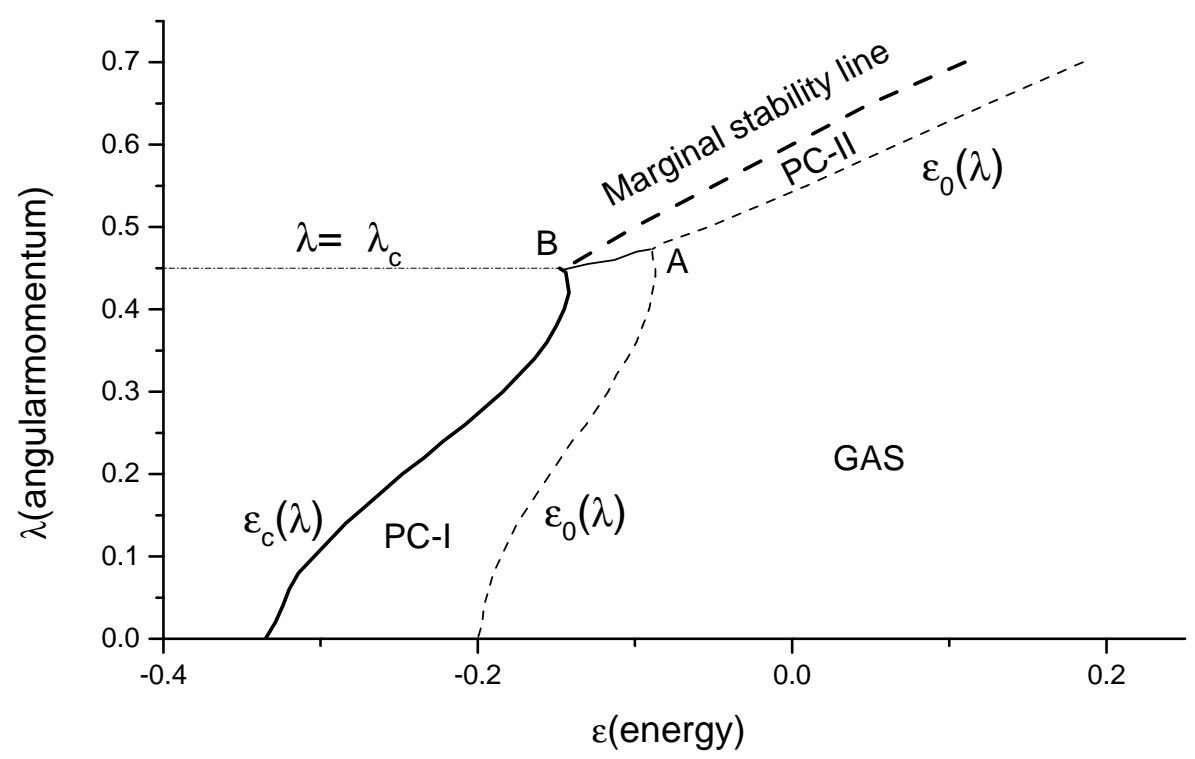

Fig. 1. Microcanonical phase diagram. GAS (axially symmetric) denotes the pure phase; phase coexistence regions with negative specific heat are denoted by PC-I (single-cluster plus "gas") and PC-II (double-cluster plus "gas"). Lines are as follows. Solid-continuous: Antonov line $\epsilon_{c}(\lambda)$, Eq. (40) . Light-dashed: phase boundary $\epsilon_{o}(\lambda)$, Eq. (42). The continuous line from A to B marks the beginning of the bifurcation line for the onset of double cluster solutions and separates the PC-I and PC-II phases; this line merges with $\epsilon_{0}(\lambda)$ right of $A$. Heavy-dashed: marginal stability line for the double cluster solution. Results have been obtained with $l_{\max }=12$.

to looking for the marginal stability line defined by the condition

$$
\left[\frac{\delta^{2} S}{\delta f(\boldsymbol{x}) \delta f\left(\boldsymbol{x}^{\prime}\right)}\right]_{G\left[f^{*}\right]=0}=0
$$

where

$$
G[f]=f(\boldsymbol{x})-\exp \left[\beta \int \frac{f\left(\boldsymbol{x}^{\prime}\right)}{\left|\boldsymbol{x}-\boldsymbol{x}^{\prime}\right|} d \boldsymbol{x}^{\prime}+\frac{\beta}{2}(\boldsymbol{\omega} \times \boldsymbol{x})^{2}+\mu\right]
$$

and $f^{*}$ is an axially asymmetric solution of $(23)$. The marginal stability line marks the border between the region of the phase diagram where axially asymmetric solution are stable from that where they are at most metastable (saddle points in the entropy surface). In principle, at the most general level the local stability analysis requires solving the eigenvalue problem for the kernel $K\left(\boldsymbol{x}, \boldsymbol{x}^{\prime}\right)=\frac{\delta^{2} S}{\delta f(\boldsymbol{x}) \delta f\left(\boldsymbol{x}^{\prime}\right)}$. We have not been able to carry out this task. However, it is possible to get numerical information about (45). Some technical details about this problem are given in [16], and we will not discuss them here. 


\subsection{Phase diagram}

The phase diagram resulting from numerical solution of (23) is displayed in Fig. 1. In Fig. 2 we show instead a small sample of equilibrium density profiles. The situation for sufficiently low angular $\lambda$ resembles that of non-rotating systems. At high $\epsilon$, a pure "gas" phase is found, where the equilibrium density profiles are axially-symmetric and particles are uniformly spread over $V$. As $\epsilon$ is decreased, the Newtonian contribution becomes more and more important and at the $\epsilon_{0}(\lambda)$ line, the specific heat becomes negative and one enters a phase coexistence region, labeled PC-I, where a collapsed configuration (see Fig. 2a) competes with the gas state (see Fig. 2b). Upon further decreasing $\epsilon$ no more solutions of (23) are found and the Antonov limit is reached. In this region, we indeed find that

$$
\lim _{\epsilon \downarrow \epsilon_{c}(\lambda)} \operatorname{Hes}(\epsilon, \lambda)=-\infty
$$

which, comparing with (40), implies that $\kappa_{2}<0$. Rotation has the only effects of shifting both the onset of negative heat capacity and the Antonov limit to higher $\epsilon$ 's (as also argued in [14]), and of deforming the density profile (as clear from Fig. 2b).

When $\lambda$ becomes larger than the critical value $\lambda_{c} \simeq 0.45$, however, the picture changes drastically. Here axially-symmetric solutions become unstable against fluctuations that break such a rotational symmetry and axially-asymmetric solutions, i.e. "double stars", occur (see Fig. 2c). The picture is clear for high $\lambda$. Upon decreasing $\epsilon$, one encounters the $\epsilon_{0}(\lambda)$ line (which in that region coincides with the bifurcation line) and enters a phase coexistence region (labeled PC-II) with negative specific heat $c_{\beta \omega}$, Eq. (43)), where double-cluster and gas configurations compete. However, at odds with the low- $\lambda$ case, a further de-
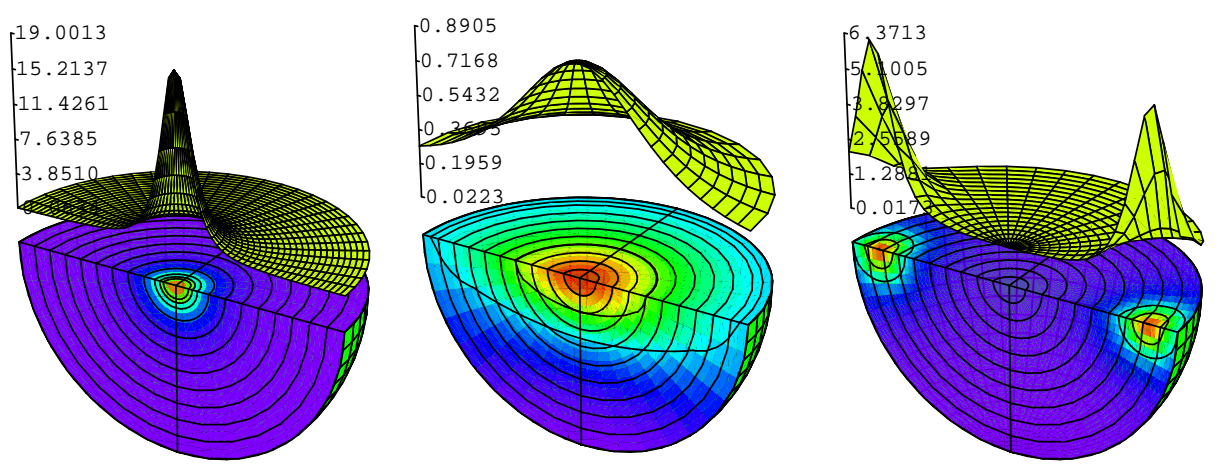

Fig. 2. Example of stable equilibrium configurations, contour plots and (above) density profiles. Left to right: single-cluster (collapsed state, $\epsilon=-0.33, \lambda=0.001$ ); "disk" $(\epsilon=-0.1, \lambda=0.4)$; double-cluster $(\epsilon=0, \lambda=0.6)$. 
crease in $\epsilon$ makes double-clusters unstable. Remarkably, the solution for lower $\epsilon$ still exists, hence there is no Antonov limit for $\lambda>\lambda_{c}$ strictly speaking. This issue will be further discussed in the next section.

The bifurcation line and the $\epsilon_{0}(\lambda)$ line do not coincide in a small range close to $\lambda_{c}$ (the $\mathrm{AB}$ line in Fig. 1 gives the bifurcation line in this range). Here the bifurcation line separates the two mixed phases, i.e. upon crossing it by increasing $\lambda$ at fixed $\epsilon$ double clusters are obtained. Transitions from PC-I to PC-II can also occur upon decreasing $\epsilon$ at fixed $\lambda$.

It is important to stress that these results were obtained by truncating the potential via $(36)$ to order $l_{\max }=12$. While calculations performed with smaller $l_{\text {max }}$ in the lower half of the phase diagram $\left(\lambda<\lambda_{c}\right)$ gave essentially the same results, for high angular momenta we observed some dependence on $l_{\max }$, albeit weak. In particular, it is possible that upon increasing $l_{\max }$ the marginal stability line is further inclined towards the $\epsilon_{0}(\lambda)$ line. As a consequence, the PC-II phase would become thinner and thinner, so that double stars would be stable (if at all) only in a very tiny portion of the parameter space. This quantitative issue, which is strictly related to the use of Boltzmann statistics, certainly deserves further investigation. We believe that no other main (qualitative) changes to the picture presented here should occur.

Finally, moving on to thermodynamics, in Fig. 3 we show the caloric curves $\beta$ vs $\epsilon$ at fixed low and, respectively, high $\lambda$. The peak in (b) corresponds to the bifurcation line.

\section{Comparison with Lynden-Bell statistics}

It is interesting to compare the behaviour of the self-gravitating and rotating gas of Boltzmann particles described above with that of a same system of Lynden Bell particles [15, 16]. Using Lynden-Bell statistics [27] instead of Boltzmann's amounts to postulating a maximum capacity of $n_{0} \ll N$ particles for each of the $K$ cells in which $V$ is subdivided in order to compute the integral (12), so that Eq. (16) would become

$$
P[\rho] \propto \frac{N !}{n\left(\boldsymbol{r}_{1}\right) ! \cdots n\left(\boldsymbol{r}_{K}\right) !} \prod_{\text {cells } k} \frac{n_{0} !}{\left(n_{0}-n\left(\boldsymbol{r}_{k}\right)\right) !}
$$

From the physical viewpoint, Lynden-Bell systems are leptodermous. This is not possible for Boltzmann particles, as seen by the fact that the clusters in Fig. 2 are sharply peaked, with the density decreasing smoothly from the maximum (Lynden-Bell particles can form stars with a dense, finite core and a fast-decreasing density [15]). Correspondingly, the stationary point condition 


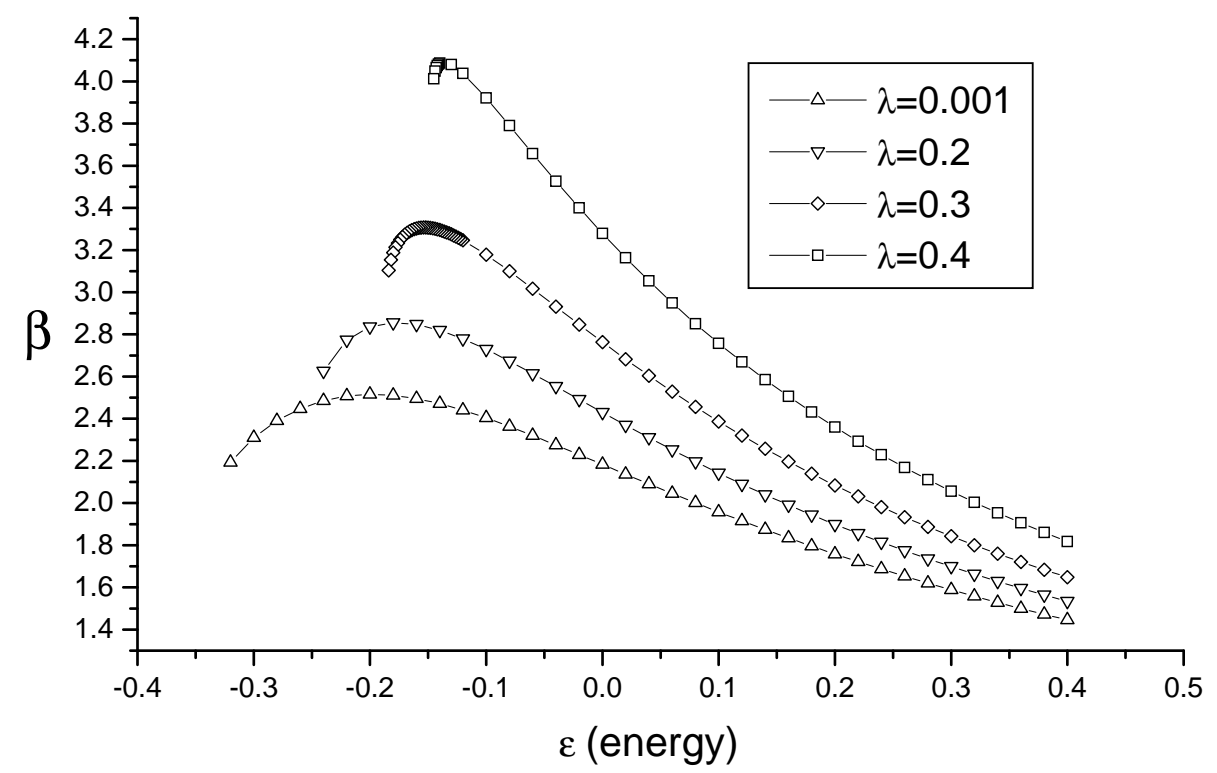

(a)

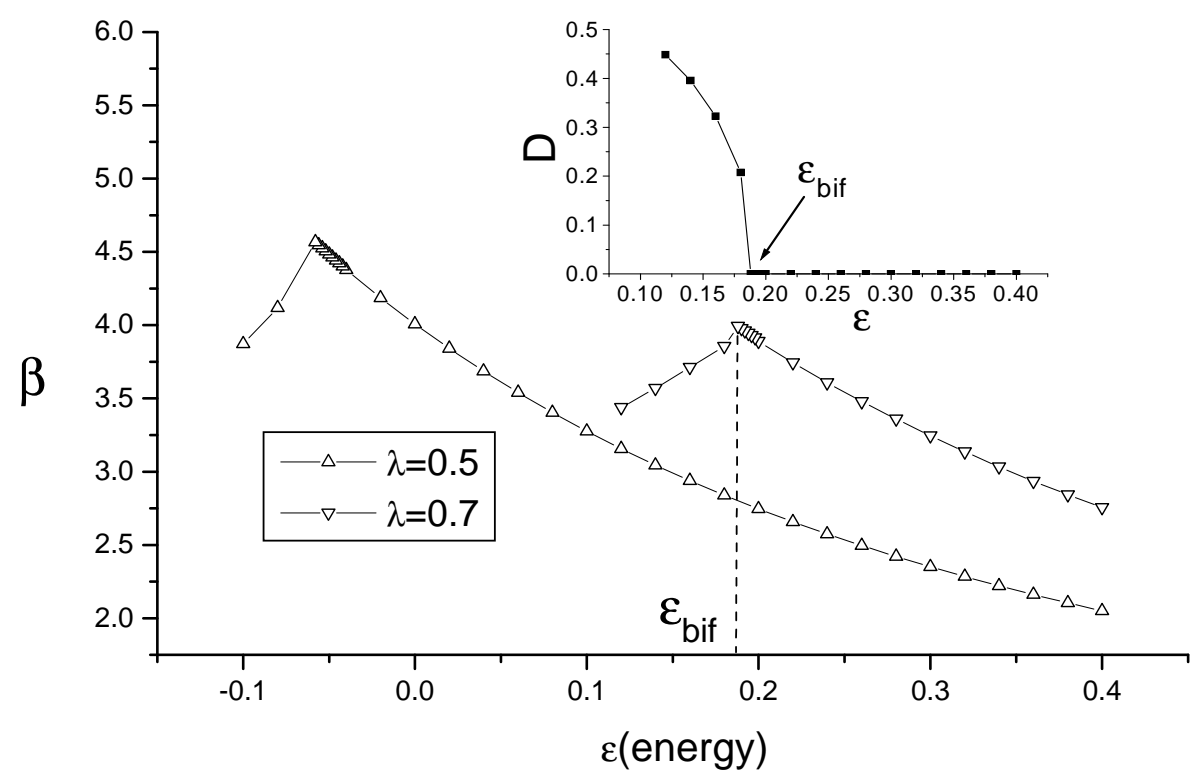

(b)

Fig. 3. Caloric curves for (a) low and (b) high $\lambda$. The inset in (b) shows the behaviour of the order parameter $D$ at $\lambda=0.7$. $\epsilon_{\text {bif }}$ in (b) denotes the bifurcation energy at $\lambda=0.7$. 
for $c=\Theta f$ reads

$$
c(\boldsymbol{x})=\left[1+\exp \left[-\frac{\beta}{\Theta} \int \frac{c\left(\boldsymbol{x}^{\prime}\right)}{\left|\boldsymbol{x}-\boldsymbol{x}^{\prime}\right|} d \boldsymbol{x}^{\prime}-\frac{1}{2} \beta(\boldsymbol{\omega} \times \boldsymbol{x})^{2}+\mu\right]\right]^{-1}
$$

This case has been studied thoroughly in $[15,16]$. Notice that, at odds with what happens in Boltzmann statistics (which is the $n_{0} \rightarrow N$ followed by $N \rightarrow \infty$ limit of the Lynden-Bell case), here the result depends on $\Theta$, which is now given by $\Theta=\frac{N V}{n_{0} K R^{3}}$, and is related to the total mass of the system.

The microcanonical phase diagram for this case is discussed in $[15,16]$, where it is shown that the system has three pure phases ("gas", single cluster and double-cluster) separated by a large phase coexistence region with negative specific heat. For historical reasons, in this paper we shall discuss the canonical phase diagram, which can be obtained by mapping the $(\epsilon, \lambda)$ variables onto their conjugate intensive variables $(T \equiv 1 / \beta, \omega)$. Evidently, in the canonical ensemble phase coexistence regions cannot be detected [17], as one must always have positive specific heat. This means that the collapse temperature is determined by the value of $\beta$ at which the derivative of $\beta$ versus $\epsilon$ changes sign, and the region PC-I, corresponding to lower energies, is not accessible. For the present comparison purposes, however, this limited information is sufficient. Canonical phase diagrams are shown in Fig. 4.

In the Boltzmann case, one sees that for high temperatures the equilibrium state is an axially-symmetric, gas-like one. At a critical temperature $T_{c}(\omega)$ the system collapses into a single cluster (for $\omega<\omega_{c} \simeq 1.03$ ) or into a double cluster (for $\omega>\omega_{c}$ ) and lower temperatures are forbidden. (For $\omega=0$ the known value $T_{c}(0) \simeq 0.3967$ is recovered.) Lynden-Bell statistics turns out to shift the onset of both the collapse to a single cluster and the formation of the double cluster to lower temperatures. However, at lower temperatures the system can exist in a single-cluster state (low $\omega$ ) or in a double cluster state (high $\omega$ ). Notice how in both cases the phase coexistence regions with negative specific heat that are found in the microcanonical phase diagrams are lost in the canonical setup.

Nevertheless, the behaviour of the Boltzmann system beyond the marginal stability line is quite intriguing. As we said, Eq. (23) admits double-cluster type of solutions for all $\epsilon$ if $\lambda>\lambda_{c} \simeq 0.45$, so there is no Antonov limit. However, such solutions are locally unstable: there exist perturbations of the density profile which lead to an increase of entropy. A profile of the entropy density $S$ versus $\epsilon$ at fixed $\lambda=0.46$ is shown in Fig. 5. At high energy the system is found in a stable, axially symmetric state: a "gas". Upon decreasing $\epsilon$, the specific heat $c_{\beta \omega}$ (43) stays positive until point $\mathrm{C}$ is reached, where it turns negative (the curvature $\kappa_{1}$ of $S(\epsilon, \lambda)$ or equivalently the quantity $\operatorname{Hes}(\epsilon, \lambda)$ is zero). One enters the phase coexistence region where collapsed configurations are possible. A further (small) decrease in $\epsilon$ will drive us to the 


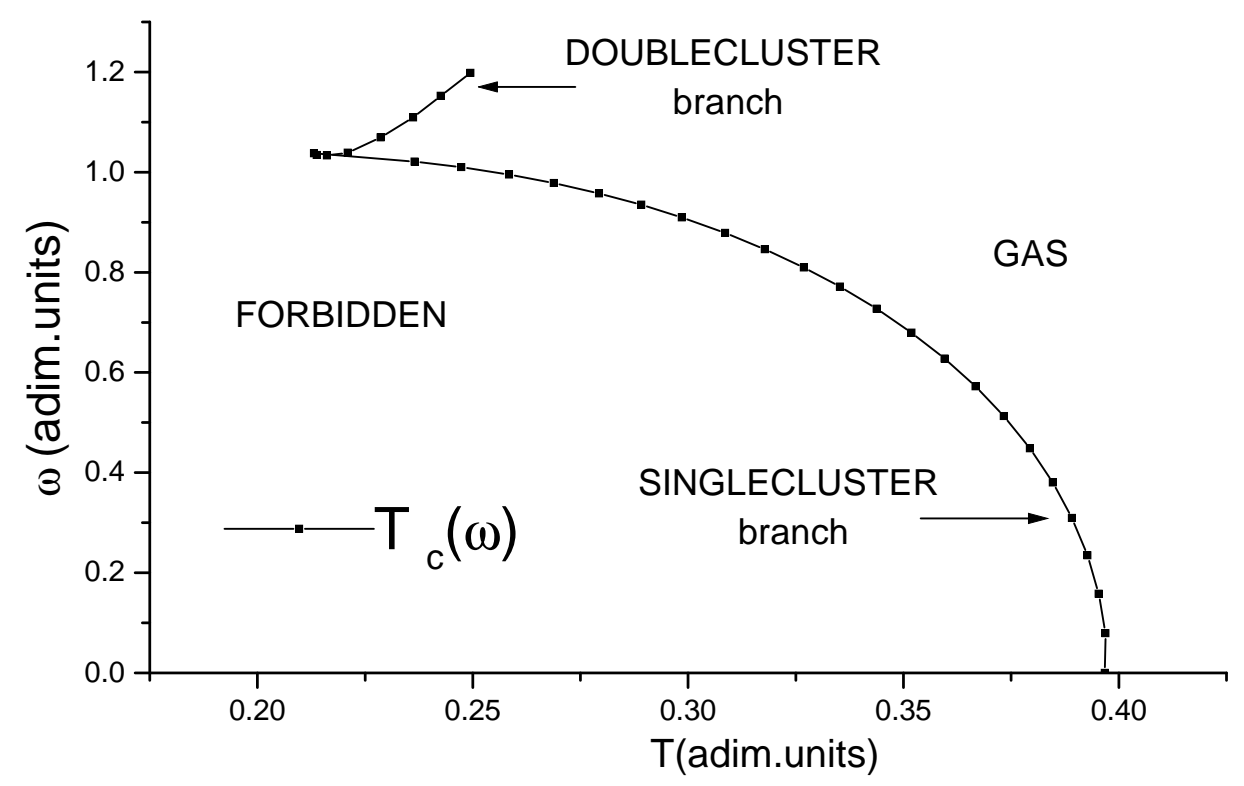

(a)

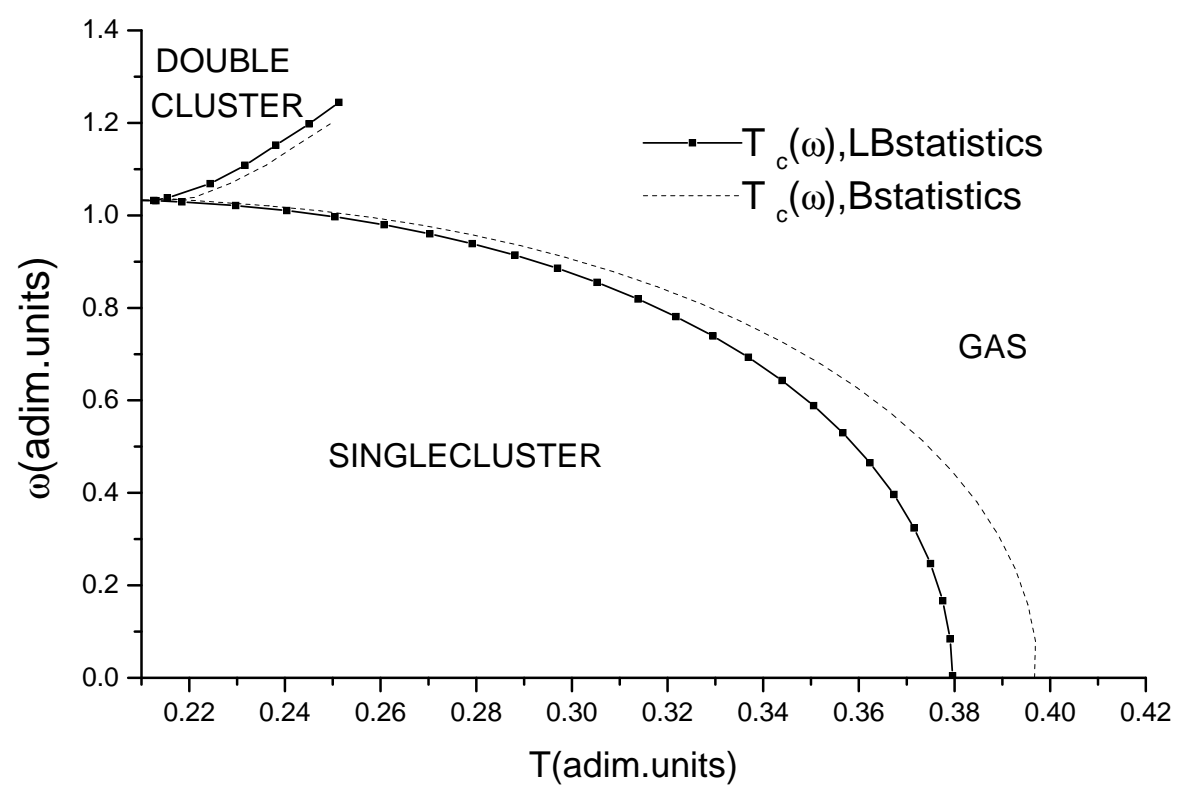

(b)

Fig. 4. Canonical phase diagram for (a) Boltzmann statistics and (b) Lynden-Bell statistics. The dashed line in (b) reproduces (a) and shows the difference between the two types of statistics. 


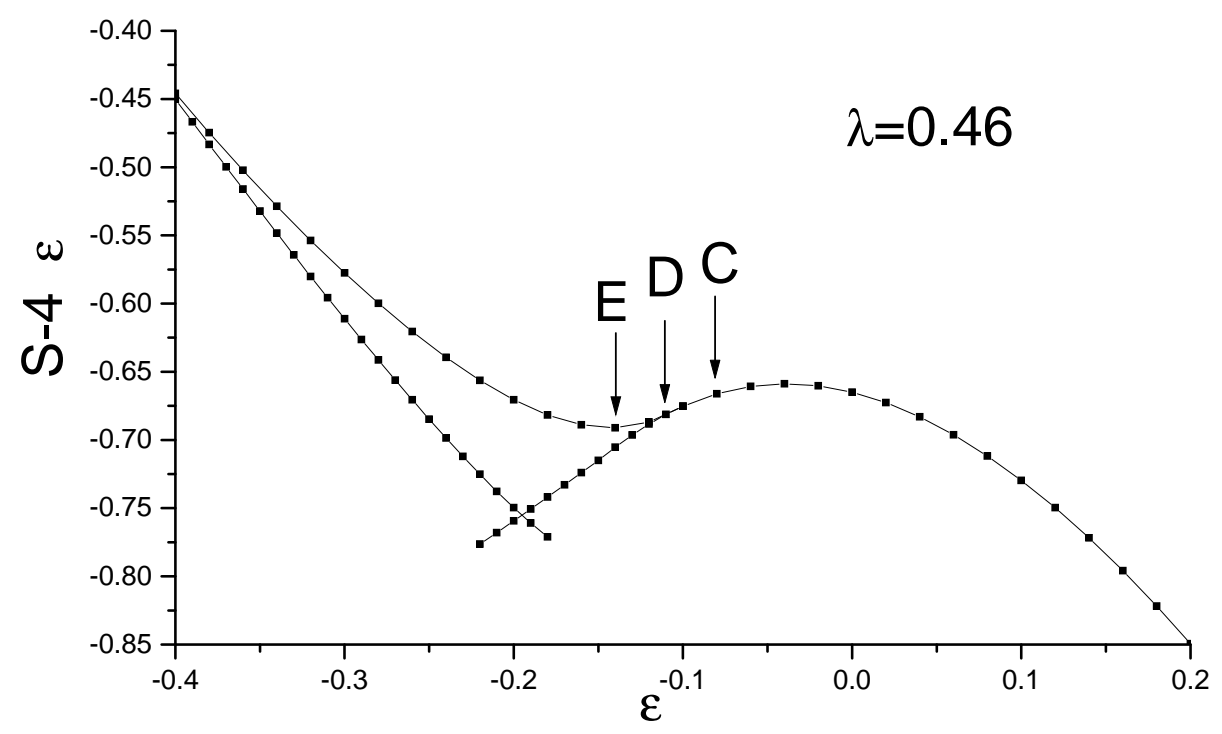

Fig. 5. (Entropy profile as a function of $\epsilon$ at fixed $\lambda=0.46$. Low energy branches correspond to double-cluster solution, the high-energy branch to the "gas"-like equilibria. Notice that $\mathrm{C}$ is a maximum of $S(\epsilon)$.

bifurcation point D, where axial-rotational symmetry breaks down in favor of such structures as "binary stars". (The axially symmetric solution however persists, though locally unstable.) Decreasing $\epsilon$ again one arrives at E, where double clusters become locally unstable, that is a saddle point of $S[f]$. Double cluster solutions (possibly more than one) however still exist left of E. In such states the two "stars" are extremely dense and almost point-like.

\section{Conclusion and outlook}

In summary, we have studied the equilibrium properties of a self-gravitating and rotating gas assuming that particles obey Boltzmann statistics in space. The physical properties of this system can be understood easily as deriving from the balance of (a) kinetic and gravitational energy at low angular momentum, and (b) gravitational and rotational energy at high angular momenta. In case (a), the effects of rotation are basically negligible except for trivial distortions of the equilibrium shapes. If the total energy is sufficiently high so that the kinetic term dominates the gravitational one, the equilibrium state of the system is axially-symmetric and gas-like. At low energies, instead, gravitation induces the system to collapse into a dense core in a low-density gas. In case (b), the high energy regime is similar but at low energy rotation is strong enough to hinder the collapse into a single cluster and drive the system to organize into a double cluster structure (a "binary star"). This simple, intuitive picture is confirmed entirely by our mean-field theory, which shows that 
in case (b) at least one main bifurcation (from axially symmetric to axially asymmetric) in the solutions of the basic Eq. (23) occurs, and possibly many others can be found upon varying the conserved quantities $\epsilon$ and $\lambda$.

Our work leaves several directions for further research. The main question concerns probably the dynamics of these systems, which is an equally old [33] and widely-discussed theme in astrophysics, see e.g. [34,35]. Among the many open issues and possible extensions for the equilibrium case (such as the effect of different masses, see $[36,37]$ ), we want to put forward the following few. First is the behaviour of the system left of the marginal stability line where double cluster solutions become unstable. (We mentioned in Sec. 3 that the location of this line turns out to depend on the maximum order of harmonics included in the calculation of the gravitational potential, $l_{\max }$. Also the fact that no Antonov limit exists for $\lambda>\lambda_{c}$ could be an effect of truncation.) At low angular momenta, namely below Antonov limit, no statistical equilibrium state exists and dynamical methods should be used to grasp the physics of the system (see e.g. [5] and references therein). But for $\lambda>\lambda_{c}$ it is still possible to obtain (unstable) equilibrium states as saddle point of the entropy surface. This means that certain perturbations applied to such "singular" double-cluster structures (highly concentrated) will lead to an increase of entropy. It is not clear to us whether local entropy maxima (metastable states) exist in this region. Definitely, there are no global maxima for $S$ is everywhere unbounded. This issue certainly deserves to be studied further. Another important question that we didn't explore concerns the relevance of mass as a further conserved quantity. If $N$ is fixed, it should be treated on the same level as $\epsilon$ and $\lambda$, and this could lead to some qualitative changes in the picture presented here. As a final consideration, we stress the mean-field character of our solution. In spite of several claims being made of mean-field theory being exact for self-gravitating systems, we believe that it would be important to investigate the effects of particle-particle correlations, also at the merely quantitative level.

Acknowledgments. Useful discussions with O. Fliegans are gratefully acknowledged.

\section{References}

[1] V.A. Antonov. Vest. Leningrad Univ. 7135 (1962).

[2] D. Lynden-Bell and R. Wood. Mon. Not. R. Astron. Soc. 138495 (1968)

[3] T. Padmanabhan. Astrophys. J. Suppl. Ser. 71651 (1989)

[4] T. Padmanabhan. Phys. Rep. 188286 (1990) 
[5] P.-H. Chavanis, C. Rosier and C. Sire. Phys. Rev. E 66036105 (2002).

[6] W. Thirring. Z. f. Phys. 235339 (1970)

[7] D. Lynden-Bell and R.M. Lynden-Bell. Mon. Not. R. Astron. Soc. 181405 (1977)

[8] J. Katz and I. Okamoto. Mon. Not. R. Astron. Soc. 317163 (2000)

[9] S. Chandrasekhar. Introduction to the study of stellar structure. Dover (New York, NY), 1939.

[10] J. Binney and S. Tremaine. Galactic dynamics. Princeton University Press (Princeton, NJ), 1987.

[11] S. Inagaki and I. Hachisu. Publ. Astron. Soc. Japan 3039 (1978)

[12] I. Hachisu. Publ. Astron. Soc. Japan 31523 (1979)

[13] O. Fliegans and D.H.E. Gross. Phys. Rev. E 65046143 (2002).

[14] P.-H. Chavanis. Astron. Astrophys. 396315 (2002).

[15] E.V. Votyakov, H.I. Hidmi, A. De Martino and D.H.E. Gross. Phys. Rev. Lett. 89031101 (2002).

[16] E.V. Votyakov, A. De Martino and D.H.E. Gross. Eur. Phys. J. B 29593 (2002).

[17] D.H.E. Gross. Microcanonical thermodynamics: Phase transitions in "Small" systems. World Scientific (Singapore), 2001.

[18] D.H.E. Gross. In T. Dauxois, S. Ruffo and E. Arimondo (Eds.), "Dynamics and thermodynamics of systems with long-range interactions", pages 23-47, Springer (Berlin), 2002.

[19] G. Gallavotti. Statistical Mechanics. Springer (Berlin), 1999.

[20] D.H.E. Gross and H. Massmann. Nucl. Phys. A 471 339c (1987)

[21] D.H.E. Gross and M. Madjet. Preprint cond-mat/9611192

[22] D.H.E. Gross and R. Heck. Phys. Lett. B 318405 (1993)

[23] P.A. Hervieux and D.H.E. Gross. Z. Phys. D 33295 (1995)

[24] D. Lynden-Bell. Physica A 263293 (1999).

[25] H.J. de Vega and N. Sanchez. Nucl. Phys. B 625409 (2002)

[26] H.J. de Vega and N. Sanchez. Nucl. Phys. B 625460 (2002)

[27] D. Lynden-Bell. Mon. Not. R. Astron. Soc. 136101 (1967)

[28] V. Laliena. Phys. Rev. E 594786 (1999).

[29] S. Chandrasekhar. Mon. Not. R. Astron. Soc. 93390 (1933) 
[30] J.D. Jackson. Classical electrodynamics. Wiley Inc. (New York, NY), 1975.

[31] H.W. Wyld. Mathematical methods for physicists. Benjamin Inc. (Reading, MA), 1976.

[32] D.H.E. Gross. Phys. Chem. Chem. Phys. 4863 (2002).

[33] M. Hénon. Annales d'Astrophysique 24379 (1961)

[34] D. Lynden-Bell and P.P. Eggleton. Mon. Not. R. Astron. Soc. 191483 (1980)

[35] H. Cohn. In "Proceedings of the 126th IAU Symposium", pages 379-392, Kluwer Academic Publishers (Dordrecht), 1988.

[36] M. Yoshizawa, S. Inagaki, M.T. Nishida, S. Kato, Y. Tanaka, Y. Watanabe. Publ. Astron. Soc. Japan 30279 (1978)

[37] H.J. de Vega and J.A. Siebert. Phys. Rev. E 66016112 (2002) 